\title{
TERNARY BOOLEAN ALGEBRA
}

\section{A. A. GRAU}

1. Introduction. The present paper ${ }^{1}$ is concerned with a ternary operation in Boolean algebra. We assume a degree of familiarity with the latter $[1,2],{ }^{2}$ and by the former we shall mean simply a function of three variables defined for elements of a set $K$ whose values are also in $K$. Ternary operations have been discussed in groupoids [4] and groups [3]; in Boolean algebra an operation different from the one introduced here was discussed by Whiteman [5].

By a simple set of postulates ( $\$ 2$ ), we define a ternary system, which we call a ternary Boolean algebra, from which Boolean algebras are obtained by specialization of the ternary operation, and which itself may be considered as a more general binary system with as many binary operations as elements, each having the properties of the Boolean operations ( $\$ 4)$. The ternary Boolean algebra is homogeneous and there is a one-to-one correspondence between distinct ternary algebras and abstract Boolean algebras $(\$ 5, \S 7)$; thus the ternary algebra provides a new postulational approach to Boolean algebra. The ternary operation has a unique realization in Boolean algebra (\$6). Other applications of ternary operations and the matter of a valid representation for ternary Boolean algebra are left to a subsequent paper.

2. Postulates for ternary Boolean algebra. Let $K$ be a system consisting of a set of elements $a, b, \cdots$, and two operations under which the system is closed, one ternary, $a^{b} c$, and the other unitary $a^{\prime}$. These satisfy the following relations for all $a, b, c, d$, and $e$ :

$$
\begin{aligned}
a^{b}\left(c^{d} e\right) & =\left(a^{b} c\right)^{d}\left(a^{b} e\right), \\
a^{b} b & =b^{b} a=b, \\
a^{b} b^{\prime} & =b^{\prime b} a=a .
\end{aligned}
$$

The system thus defined we shall call a ternary Boolean algebra.

It is easily verified that the following function in Boolean algebra satisfies the postulates:

Received by the editors October 28, 1946,

1 This paper is a portion of a dissertation submitted in partial fulfillment of the requirements for the degree of Doctor of Philosophy in the University of Michigan, May 1944.

${ }^{2}$ Numbers in brackets refer to the bibliography at the end of the paper. 


$$
(a \cap b) \cup(b \cap c) \cup(c \cap a) .
$$

The system $K$ possesses a realization therefore and the postulates are consistent. By specifying zero and unit elements, 0 and 1 , and then restricting the ternary product $a^{b} c$ to $a \cap b=a^{0} b$ and $a \cup b=a^{1} b$, Boolean algebra arises as a special case of ternary Boolean algebra (cf. §4).

The principal property of $K$ is expressed by postulate (2.1); it is what we shall call distributive. The other two postulates are reducibility conditions, which state that under certain conditions the ternary product becomes a function of only one of the three elements involved. They are analogous to the identity and absorption relations in binary Boolean algebra.

The propositions of Boolean algebra exhibit a duality in the operations. In the ternary algebra this is replaced by homogeneity; we do not have elements with special significance such as 0 and 1 . The fact that $K$ is homogeneous (that is, that all elements have equivalent properties) will be discussed in detail later.

3. Theorems in ternary Boolean algebra. A few basic theorems that we require are proved here; the proofs are for the most part analogous to those of the corresponding theorems in Boolean algebra.

Theorem 3.1. For each element $b$ the element $b^{\prime}$ is unique.

For if for some $b$ there were two distinct complements $b_{1}^{\prime}$ and $b_{2}^{\prime}$ we have by postulate (2.3):

$$
b_{1}^{\prime}=b_{1}^{\prime}{ }^{b} b_{2}^{\prime}=b_{2}^{\prime} .
$$

THEOREM 3.2. The idempotent law holds, $a^{b} a=a$.

$$
\begin{aligned}
a^{b} a & =\left(a^{b} b^{\prime}\right)^{b}\left(a^{b} b^{\prime}\right) \\
& =a^{b}\left(b^{\prime b} b^{\prime}\right) \\
& =a^{b} b^{\prime} \\
& =a
\end{aligned}
$$

THEOREM 3.3. The associative law, $a^{b}\left(c^{b} d\right)=\left(a^{b} c\right)^{b} d$, holds for any derived binary operation $b$.

The proof requires a lemma, $\left(a^{b} c\right)^{b} a=a^{b} c$ :

$$
\begin{aligned}
\left(a^{b} c\right)^{b} a & =\left(a^{b} c\right)^{b}\left(a^{b} b^{\prime}\right)=a^{b}\left(c^{b} b^{\prime}\right) \\
& =a^{b} c .
\end{aligned}
$$

Then the theorem follows by: 


$$
\begin{aligned}
a^{b}\left(c^{b} d\right) & =\left(a^{b} c\right)^{b}\left(a^{b} d\right) \\
& =\left[\left(a^{b} c\right)^{b} a\right]^{b}\left[\left(a^{b} c\right)^{b} d\right] \\
& =\left(a^{b} c\right)^{b}\left[\left(a^{b} c\right)^{b} d\right] \\
& =\left[\left(a^{b} c\right)^{b} b^{\prime}\right]^{b}\left[\left(a^{b} c\right)^{b} d\right] \\
& =\left(a^{b} c\right)^{b} d
\end{aligned}
$$

THEOREM 3.4. $\left(a^{\prime}\right)^{\prime}=a$.

We use the lemma $a^{b^{\prime}} b=a$ :

$$
a^{b^{\prime}} b=\left(a^{b} b^{\prime}\right)^{b^{\prime}}\left(a^{b} b\right)=a^{b}\left(b^{\prime b^{\prime}} b\right)=a .
$$

The theorem may be proved using (2.3):

$$
\left(a^{\prime}\right)^{\prime}=\left(a^{\prime}\right)^{\prime a^{\prime}} a=a .
$$

THEOREM 3.5. $a^{b} a^{\prime}=b$.

$$
\begin{aligned}
a^{b} a^{\prime} & =a^{b}\left(b^{a^{\prime}} a^{\prime}\right) \\
& =\left(a^{b} b\right)^{a^{\prime}}\left(a^{b} a^{\prime}\right) \\
& =b^{a^{\prime}}\left(a^{b} a^{\prime}\right) \\
& =\left(b^{a^{\prime}} a\right)^{b}\left(b^{a^{\prime}} a^{\prime}\right) \\
& =b^{b}\left(b^{a^{\prime}} a^{\prime}\right) \\
& =b
\end{aligned}
$$

THEOREM 3.6. The operation $a^{b} c$ is commutative in any pair of elements.

(a)

$$
\begin{aligned}
a^{b} c & =a^{b}\left(a^{c} a^{\prime}\right) \\
& =\left(a^{b} a\right)^{c}\left(a^{b} a^{\prime}\right) \\
& =a^{c} b \\
a^{b} c & =a^{b}\left(b^{c} b^{\prime}\right) \\
& =\left(a^{b} b\right)^{c}\left(a^{b} b^{\prime}\right) \\
& =b^{c} a
\end{aligned}
$$

(c) $a^{b} c=c^{b} a$. This follows from the propositions (a) and (b).

We shall call a ternary operation in which each pair of elements may be interchanged without changing its value completely commutative. If one pair of elements may be interchanged, but the others may not, then we have partial commutativity.

THEOREM 3.7. For any $x$ of $K$,

$$
a^{b} c=\left(a^{x} b\right)^{x^{\prime}}\left(b^{x} c\right)^{x^{\prime}}\left(c^{x} a\right) .
$$


Proof.

$$
\begin{aligned}
a^{b} c & =a^{b}\left(x^{c} x^{\prime}\right) \\
& =\left(a^{b} x\right)^{c}\left(a^{b} x^{\prime}\right) \\
& =\left(a^{x} b\right)^{c}\left(a^{x^{\prime}} b\right) \\
& =\left[\left(a^{x} b\right)^{c}\left(a^{x} x^{\prime}\right)\right]^{x^{\prime}}\left[\left(a^{x} b\right)^{c}\left(x^{\prime} x b\right)\right] \\
& =\left[a^{x}\left(b^{c} x^{\prime}\right)\right]^{x^{\prime}}\left[\left(a^{c} x^{\prime}\right)^{x} b\right] \\
& =\left[\left(a^{x} b\right)^{x^{\prime}}\left(a^{x} c\right)\right] x^{x^{\prime}}\left[\left(a^{x} b\right)^{x^{\prime}}\left(c^{x} b\right)\right] \\
& =\left(a^{x} b\right)^{x^{\prime}}\left(b^{x} c\right)^{x^{\prime}\left(c^{x} a\right)}
\end{aligned}
$$

4. Associated Boolean algebras. Let $p$ be a fixed element of $K$. Define

$$
a \cap b=a^{p} b, \quad a \cup b=a^{p^{\prime}} b
$$

and refer to the system formed by the elements of $K$ and the operations $\cap$ and $\cup$ as $B(p)$. We may prove:

THEOREM I. The system $B(p)$ forms a Boolean algebra with $p$ as its universe element and $p^{\prime}$ as its null element.

The operations $\cap$ and $\cup$ are commutative by (3.6), associative by (3.3), and distributive by (2.1). That $p$ and $p^{\prime}$ are respectively the identities of multiplication and addition is easily verified by (2.3), and complementary elements satisfy the relations (3.5). Thus the system $B(p)$ has the properties of a Boolean algebra.

Theorem I enables us to translate theorems of Boolean algebra into theorems in $K$. For if we have a theorem in Boolean algebra, for example, deMorgan's theorem:

$$
(a \cap b)^{\prime}=a^{\prime} \cup b^{\prime},
$$

it must hold in $B(p)$ since the latter is a Boolean algebra. But by (4.1) this becomes

$$
\left(a^{p} b\right)^{\prime}=a^{\prime p^{\prime}} b^{\prime},
$$

for any $p$ of $K$, and so we may state the following theorem.

ThEOREM 4.3. $K$ possesses the deMorgan property (4.3).

It will be noted that deMorgan's theorem takes a symmetric form in $K$ and expresses the fact that the unitary operation is distributive over the ternary operation $a^{b} c$. In order to find the complement of an expression in $K$ it is necessary only to put primes on the unprimed letters and unprime the primed ones. 
Theorem I allows a further observation. For a fixed $b$, the ternary product $a^{b} c$ is a binary operation which has the properties of a Boolean binary operation. Since for each $b$ there is such an operation, $K$ may also be considered as a binary system in which there are as many Boolean operations as elements, and every pair of these operations satisfy the Boolean distributive law.

5. Automorphisms of the ternary Boolean algebra. We stated that $K$ is homogeneous in its elements, meaning that every element of $K$ has the same properties as any other. This fact will now be expressed in a concrete form in terms of automorphisms.

A function $f(x)$ is an automorphism of $K$ if $f(x)$ is a one-to-one transformation of $K$ on to itself leaving invariant the ternary operation and the operation of complementation. If the former is preserved, so is the latter; for if $f(x)$ preserves the ternary operation,

$$
f(x)=f\left(x^{y} y^{\prime}\right)=f(x)^{f(y)} f\left(y^{\prime}\right)
$$

for all $x$ and $y$. If $x, y$, and $y^{\prime}$ are distinct, so are $f(x), f(y)$, and $f\left(y^{\prime}\right)$. By (2.3) and (3.1) it follows therefore that $[f(y)]^{\prime}=f\left(y^{\prime}\right)$.

The function below will be shown by a sequence of lemmas to express an automorphism of $K$ transforming $a$ into $b$,

$$
f(x)=\left(b^{\prime a} x\right)^{a^{\prime}}\left(b^{a} x^{\prime}\right) .
$$

LEMMA 1. $f(a)=b$.

This follows immediately by substitution.

LEMMA 2. $f(x)^{a} f(y)=\left(b^{\prime a} x^{a} y\right)^{a^{\prime}}\left(b^{a} x^{\prime a} y^{\prime}\right)$.

This follows from the definition of $f(x)$ and the distributive law.

LEMMA 3. $f\left(x^{y} z\right)=f(x)^{f(y)} f(z)$.

$$
\begin{aligned}
& f\left(x^{y} z\right)=\left[b^{\prime a}\left(x^{y} z\right)\right]^{a^{\prime}}\left[b^{a}\left(x^{\prime} y^{\prime} z^{\prime}\right)\right] \\
& \quad=\left[b^{\prime a}\left\{\left(x^{a} y\right)^{a^{\prime}}\left(x^{a} z\right)^{a^{\prime}}\left(y^{a} z\right)\right\}\right]^{a^{\prime}}\left[b^{a}\left\{\left(x^{\prime a} y^{\prime}\right)^{a^{\prime}}\left(x^{\prime a} z^{\prime}\right)^{a^{\prime}}\left(y^{\prime a} z^{\prime}\right)\right\}\right]
\end{aligned}
$$

By the results of $\$ \S 2-3$, simplification, and use of Lemma 2 we obtain

$$
\begin{aligned}
f\left(x^{y} z\right) & =\left[f(x)^{a} f(y)\right]^{a^{\prime}}\left[\left(f(y)^{a} f(z)\right]^{a^{\prime}}\left[f(z)^{a} f(x)\right]\right. \\
& =f(x)^{f(y)} f(z)
\end{aligned}
$$

LEMMA 4. $f[f(x)]=x$.

This follows by direct substitution and simplification.

THEOREM II. For given $a$ and $b$, there exists an automorphism of $K$ transforming $a$ into $b$. 
Proof. By Lemma 1, the function (5.2) carries $a$ into $b$, by Lemma 3 , it leaves invariant the ternary operation and hence complementation, and by Lemma 4, it has a single-valued inverse, namely, itself.

CoROLlary. If $p$ and $q$ are any two elements of $K$, the Boolean algebras $B(p)$ and $B(q)$ are isomorphic.

It is necessary only to consider the automorphism of $K$ that transforms $p$ into $q$. This transforms the operations of $B(p)$ in to the corresponding ones of $B(q)$.

6. Realizations of the ternary algebra. We have pointed out that the function $(a \cap b) \cup(b \cap c) \cup(c \cap a)$ in Boolean algebra satisfies the postulates for the ternary algebra. It will now be shown that this is the only realization of the ternary product $a^{b} c$ in an associated Boolean algebra $B(p)$. For Theorem 3.7 states an identity in $x$; the value of $a^{b} c$ is independent of the value of $x$ and we may assign the latter at pleasure. Let $x=p$. Then in $B(p)$ we must have

$$
a^{b} c=(a \cap b) \cup(b \cap c) \cup(c \cap a) .
$$

Hence the theorem:

THEOREM III. The only realization of the ternary operation in a Boolean algebra is

$$
a^{b} c=(a \cap b) \cup(b \cap c) \cup(c \cap a) .
$$

7. Relationship of the ternary and binary algebras. We see from the preceding two paragraphs that there exists a one-to-one correspondence between a ternary Boolean algebra and an abstract Boolean algebra. Nonisomorphic Boolean algebras give rise to nonisomorphic ternary Boolean algebras and conversely.

It follows that Boolean algebra is fully characterized by the postulates (2.1)-(2.3) and the definitions (4.1).

\section{BIBLIOGRAPHY}

1. G. Birkhoff, Lattice theory, Amer. Math. Soc. Colloquium Publications, vol. 25, 1940, chap. 6.

2. G. Birkhoff and S. MacLane, $A$ survey of modern algebra, Macmillan, 1941, chap. 11.

3. J. Certaine, The ternary operation $(a b c)=a b^{-1} c$ of a group, Bull. Amer. Math. Soc. vol. 49 (1943) pp. 869-877.

4. A. R. Richardson, Algebra of $s$ dimensions, Proc. London Math. Soc. vol. 47 (1940) pp. 38-59.

5. A. L. Whiteman, Postulates for Boolean algebra in terms of ternary rejection, Bull. Amer. Math. Soc. vol. 43 (1937) pp. 293-298.

UNIVERSITY OF KeNTUCKY 